\title{
DyMoRail: A Modelica Library for modelling railway buffers
}

\author{
Elisabeth Dumont Werner Maurer \\ Zentrum für angewandte Mathematik und Physik, Zürcher Hochschule für Angewandte Wissenschaften \\ Technikumstrasse 9, Winterthur, 8401 Switzerland
}

\begin{abstract}
This article gives an overview of the DyMoRail library.The aim of this Modelica library is the simulation of longitudinal dynamics of entire railway trains. The DyMoRail library allows an efficient simulation of complete train compositions in various configurations. The library contains different car models, buffers, couplers equipped with both friction and elastomer springs, as well as the center-buffers for multiple units. DyMoRail allows to simulate the entire motion cycle that the buffer undergoes during a collision. The robust programming of the basic models allows simulations for arbitrary combination of buffers, couplers and destruction tubes. Different modelling techniques (SIMULINK, STELLA) have been explored. Since the modular structure of Modelica allows fast and simple setup of models including different types of rolling stock and different types of couplers and buffers, it was decided to build this library in Modelica. This simulation environment was successfully used by Schwab Verkehrstechnik AG during the development of their state-of the-art center coupler product family. Within DyMoRail2 we intend to implement further features and improve the modularity and flexibility of the library.

Keywords: library, mechanics, railway
\end{abstract}

\section{Introduction}

Buffers and couplers are an essential part of the railway wagon. They have to be optimized for new wagon types to work for different train compositions. They have to absorb minor impacts, take up slack between locomotive and wagons and bear the load of preceding wagons when pushing. Years ago it was good enough for couplers and buffers to fulfil UIC (International Union of Railways) standards. But nowadays manufacturers only survive in this competitive market if they are able to offer optimized solutions regarding force, energy absorption, and driving comfort. Mod- elling plays an important role in this optimization process. One of the main requirements to this rail model are that it should allow easy substitution of components and handling of different combinations of subsystem parts.

Schwab Verkehrstechnik AG and ZHAW carried out a project funded by CTI (Swiss Federal Commission for Technology and Innovation) to develop a simulation tool which allows to model longitudinal dynamics of entire railway trains. During the following years a Modelica library has been developed which is called DyMoRail. The DyMoRail library allows an efficient simulation of complete train compositions in various configurations. The library contains a number of different car models, buffers, couplers equipped both with friction and elastomer springs, as well as the center-buffers for multiple units (such as Seetalbahn, Thurbo, Flirt). DyMoRail allows to simulate the entire motion cycle during a collision (retraction of the buffer, force increase with stroke of the buffer, extension of the buffer, and finally the separation of the wagons).

The robust programming of the basic models allows for arbitrary combination of buffers, train draw rod and destruction tubes. In a first attempt, simulations were performed with SIMLULINK. But it turned out that in SIMULINK a completely new model had to be programmed from scratch for each combination. Therefore Schwab Verkehrstechnik and ZHAW decided to build a new library based on Modelica. The modular structure of Modelica allows fast modifications of the model by simple replacement of entire subsystems. In this paper we will present the structure of the existing library, show some examples and propose some improvements that will lead to a new version DyMoRail2, which will be constructed in collaboration with Schwab Verkehrstechnik AG and is funded by CTI. 


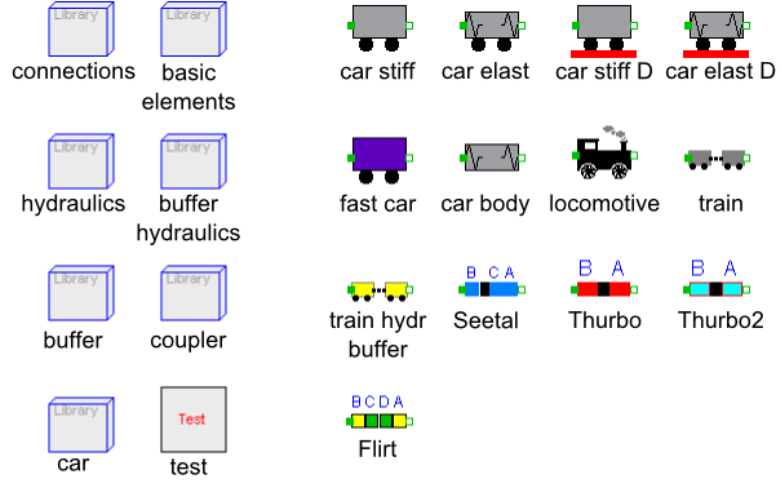

Figure 1: DyMoRail library structure

\section{Library Structure}

The DyMoRail library structure is shown in Figure 1. The fundamental packages and models are explained in the following paragraph. The library consists of seven sublibraries: connectors, basic elements, hydraulics, buffer hydraulics, buffers, couplers and cars.

\subsection{Connectors}

The sublibrary connectors contains the mechanical and hydraulic connections as well as the connections between the hydraulic buffers and the respective buffer hydraulics.

\subsection{Basic Elements}

This sublibrary contains different models of springs and buffers, as well as friction between car and railway track. The submodels "buffer bush" and "coupler bush" simulate the fundamental behaviour of the buffers and couplers. Four different operation modes of the buffer bush are distinguished: free, pretension, deformation and arrested. In free mode, the buffer plates do not touch and the force is zero. In the pretension mode the force increases. In the deformation mode the buffer spring and buffer hydraulics are loaded. In the arrested mode the force increases steeply. The additional state forward, backward and halt describe the actual condition of the bush. In addition friction is also modelled in the bush.

\subsection{Hydraulics}

In addition to the basic elements for viscous flow, this sublibrary contains hydraulic accumulator and check valves and multiplier valves for various buffers and couplers. Each multiplier valve has three signal inputs:
The first signal yields the state of the buffer. The valve opens only if the buffer state is on deformation and is not retracted. The second input provides the opening of the aperture so that oil can flow into the hydraulic buffer. The third input provides the deformation of the buffer bush.

\subsection{Buffer an Couplers}

These sublibraries contain products of the company Schwab Verkehrstechnik AG, such as buffers, couplers, coupling rods and railway compositions. Elastomer springs are commonly used, because they are cost saving and robust. They show a non linear characteristic and have high inner friction. The friction depends on the buffer force and has both a linear and a non-linear part.

The library contains a basic model for both the hydraulic buffer and coupler. The hydraulics, which have to be reconfigured for each train, are filed in the buffer hydraulics sublibrary.

\subsection{Cars}

This sublibrary (also shown in Figure 1) contains elements, which allow the modelling of cars as rigid or flexible bodies, as well as car bodies and locomotives. Two additional models allow to model freight trains with an arbitrary number of wagons. These trains are equipped with either standard buffers or hydraulic buffers. The library contains further models which describe multiple units of Stadler Rail AG (such as GTW, FLIRT, KISS).

\section{Examples}

Two different examples are presented in the following paragraph.

\section{1 $1 g$-Buffer}

The acceleration of lightly loaded freight cars during a shunting impact can reach levels as high as $40 \mathrm{~m} / \mathrm{s}^{2}$ $(4 \mathrm{~g})$. Such hard collisions mean a high risk of damage to the freight. Since for transportation by trucks much lower accelerations of the order of $0.8 \mathrm{~g}$ occur, this is a severe disadvantage of the rail transport compared with road transport. The $1 g$-buffer was developed in order to protect damageable freight during shunting impacts. This buffer should keep the maximum acceleration of the wagons below $10 \mathrm{~m} / \mathrm{s}^{2}$ at an impact velocity of $7.2 \mathrm{~km} / \mathrm{h}$. The buffer shows the same static 
behaviour as a conventional UIC-526 buffer, i.e. the force increases up to a value of $900 \mathrm{kN}$ at a stroke of $150 \mathrm{~mm}$. Under static load, the buffer can only retract by half of its length up to a maximum force of $150 \mathrm{kN}$. Due to an elaborate valve control the $1 g$-buffer is dynamically more flexible than statically. At first this buffer has been modelled with SIMULINK. However, modelling with DyMoRail allows a larger variety of different scenarios. Besides the DyMoRail model is much more precise and detailed.

In Figure 2 a model for a collision between two wagons is depicted. A freight car of $80 \mathrm{t}$, respectively $30 \mathrm{t}$, collides with a car at rest. The moving car is equipped with a $1 \mathrm{~g}$-buffer and the car at rest with standard UIC-buffers. Figure 3 and 4 show the simulation results. The force-stroke-behaviour of the $1 g$-buffer is drawn during shunting impacts. For both cases, the acceleration of the cars does not exceed $10 \mathrm{~m} / \mathrm{s}^{2}(1 \mathrm{~g}) .1 \mathrm{~g}$ buffers are used nowadays mostly for freight cars that transport road semi-trailers. According to the simulated data the $1 \mathrm{~g}$ buffer complies with DB Cargo standards.

The model has been validated with measurements performed on the $1 g$ buffer[1].

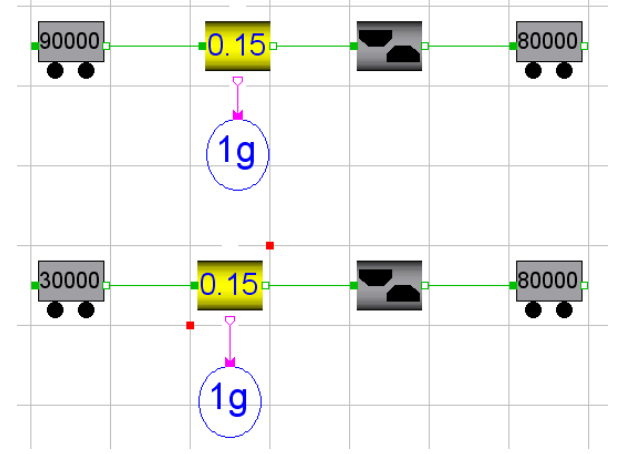

Figure 2: Model for a collision between two wagons. A freight car of $90 \mathrm{t}$, or $30 \mathrm{t}$ respectively, collides with a car at rest. The moving car is equipped with a $1 g$ buffer (yellow) and the car at rest with standard UICbuffers (grey).

\subsection{S-Bahn}

For Zurich S-Bahn trains of the third generation, multiple units consisting of 6 double decker coaches are used. A single assembly has a mass of $312 \mathrm{t}$, a total length of $150 \mathrm{~m}$ and can take up to 1694 passengers. It is obvious that, during shunting, the rolling stock must not be damaged at all. This means that the central coupler must not be damaged during a collision of such a

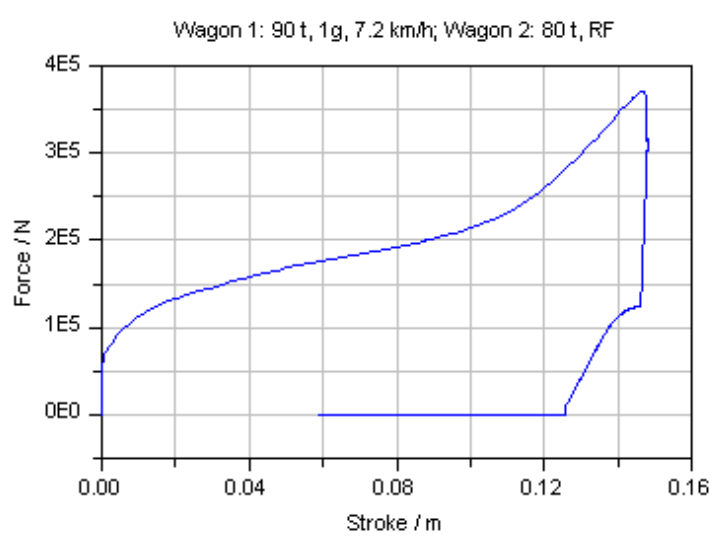

Figure 3: Force-stroke diagramm of the $1 \mathrm{~g}$ buffer during a collision of a $90 \mathrm{t}$ wagon equipped with a $1 \mathrm{~g}$ buffer at a velocity of $7.2 \mathrm{~km} / \mathrm{h}$ with a $80 \mathrm{t}$ wagon at rest equipped with standard UIC-buffers.

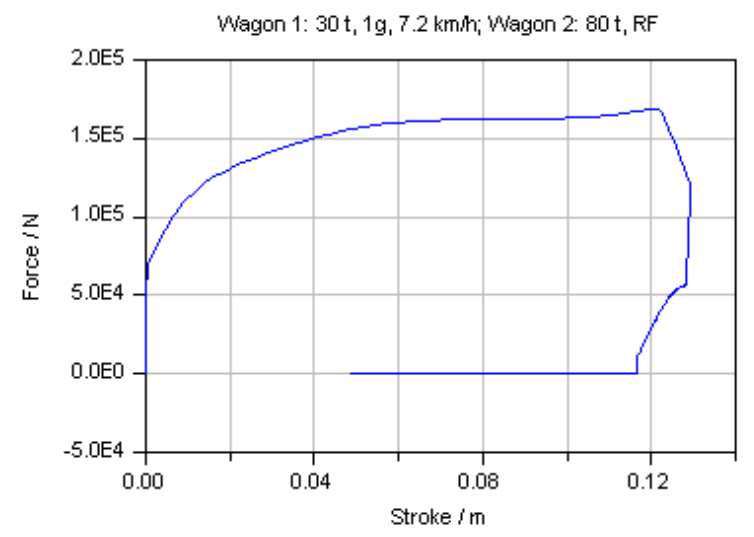

Figure 4: Force-stroke diagramm of the $1 \mathrm{~g}$ buffer during a collision of a $30 \mathrm{t}$ wagon equipped with a $1 \mathrm{~g}$ buffer at a velocity of $7.2 \mathrm{~km} / \mathrm{h}$ with a $80 \mathrm{t}$ wagon at rest equipped with standard UIC-buffers.

multiple unit at a speed of up to $5 \mathrm{~km} / \mathrm{h}$ with another one.

Furthermore it has to be proven that the coupler is pulled down correctly during a major impact with another S-Bahn up to a speed of $36 \mathrm{~km} / \mathrm{h}$ and that the side buffer are capable of absorbing the remaining energy. The same proof has to be provided for a collision at $36 \mathrm{~km} / \mathrm{h}$ against a freight car of mass $80 \mathrm{t}$. First the central coupler retracts and after that breaks away so that the laterally mounted auxiliary buffers take up the remaining energy. The entire process including pressure build-up, opening of the hydraulic predetermined breaking point, retraction of the damper, compression of the spring elements and deformation of the crash elements can be simulated in a single run.

The damper of the central coupler is a multifunc- 
tional device (Figure 6). It contains a gas spring and a multiplier valve. They ensure that the coupler transmits the momentum and secondly absorbs enough energy to prevent the wagons from oscillating during the journey. During coupling at a speed of $5 \mathrm{~km} / \mathrm{h}$ the damper has to absorb the total energy over a length of $140 \mathrm{~mm}$ without the force increasing above $1200 \mathrm{kN}$. If the the force increases above $1500 \mathrm{kN}$, a hydraulic breaking point will be activated so that the coupler is retracted faster. In addition, the coupler comes with a return stroke damping, which prevents breakaway during run-up.

Every one of these scenarios has been simulated with a dynamical model for both multiple units. Thereby the flexibility of the car body, its connection to the bogie and the behaviour of the short couplers between cars have to be modelled with sufficient precision

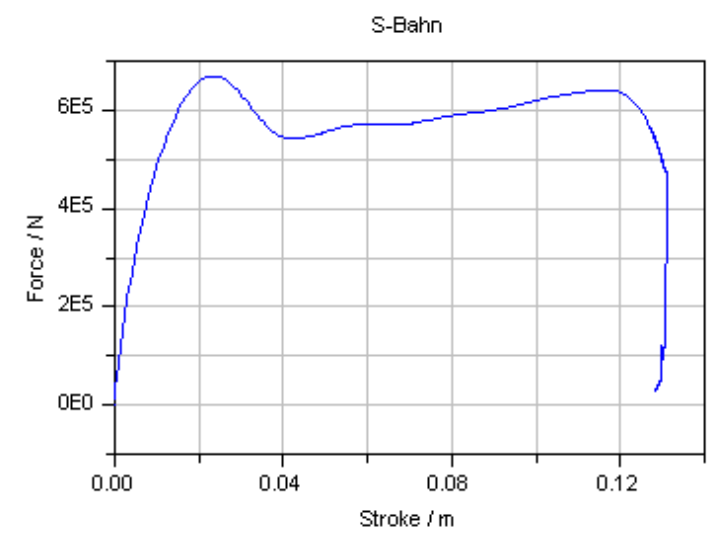

Figure 5: Force-stroke diagram for an entire S-Bahn multiple unit consisting of 6 double decker coaches with a total mass of $312 \mathrm{t}$ and a total length of $150 \mathrm{~m}$

\section{Future Work}

A follow up project (called DyMorail2) has been funded by CTI and will be carried out in collaboration with Schwab Verkehrstechnik AG. We intend to implement the following improvements to the first DyMoRail library:

1. Each buffer and coupler should be modelled in different levels of detail and complexity, in order to gain flexibility for simulating entire compositions consisting of several cars on the one hand and single wagons on the other.

2. The valve control has to be redesigned. At present the valve is modelled such that it opens

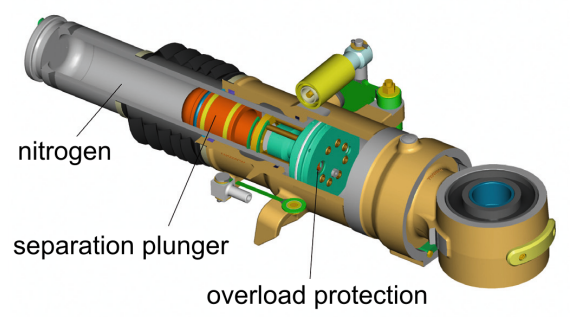

Figure 6: Construction drawing of the damper showing its working principle. It shows the damper bush in brass colour. On the rear is the air spring filled with nitrogen. The front part contains oil and both chambers are separated by a movable piston ("separation plunger" in red). In green is shown the overload pressure valve.

at a certain pressure and closes again at a lower one. This model is very simple and robust and can be used for a broad spectrum of applications. However, for modelling of long trains equipped with hydraulic buffers in combination with spring buffers these valves produce a lot of events, which increases the simulation complexity.

3. We also plan to implement crash scenarios according to new European norm DIN EN 15227. It contains requirements to the construction of rolling stock in order to minimize the consequence of collisions. It applies to the car body as well as to coupler and buffer.

\section{Conclusion}

With DyMoRail1 a powerful library has been implemented which allows to simulate longitudinal dynamics of entire railway trains. With this library an efficient simulation of complete train compositions in various combinations is possible. Modelica/Dymola has the following advantages over other tools such as SIMULINK or STELLA:

- Every model can be reused immediately

- Cars, buffers, crash elements and couplers can be arbitrarily combined

- Little effort is needed to establish, test and reconfigure new models

- Documentation and filing of simulation experiments is straight forward 
- Even non-experts can carry out simulations with DyMoRail

This simulation environment was successfully used by Schwab Verkehrstechnik AG during the development of their state-of the-art center coupler product family. Within DyMoRail2 we intend to implement further features and improve the modularity and flexibility of the library.

\section{References}

[1] Maurer W. Puffer nach Mass. Eisenbahn Revue 3, 2003, p.118-119.

[2] Maurer W. Simulationsgestütze Entwicklung von Puffern und Dämpfern für Eisenbahnzüge. Proceedings of the 18th Symposium on Simulationtechnique ASIM 2005, Erlangen, Germany, ASIM September 12-15 2005. 
